\title{
Narrativas de sostenibilidad en emprendedores sociales de Lima Metropolitana
}

\section{Narratives of sustainability in social entrepreneurs of Lima, Perú}

\author{
Angela Vera Ruiz, María Ángela Prialé Valle, Agustín Espinosa Pezzia y Erle Frances Ninahuanca López \\ Pontificia Universidad Católica del Perú \\ (Rec: abril de 2019 - Acept: octubre de 2019)
}

\begin{abstract}
Resumen
El aprecio por prácticas sostenibles (APS) es una dimensión psicológica actitudinal que la literatura actual define como componente relevante del comportamiento emprendedor social. El presente estudio busca comprender la manera en que esta dimensión se gesta y hace consistente en las narrativas de vida de 10 líderes de emprendimientos sociales de Lima Metropolitana, buscando dar fundamento vivencial a un constructo que, si bien está definido conceptualmente, no ha sido abordado desde las experiencias personales que lo hacen posible. A través de una metodología cualitativa de enfoque narrativo, y bajo una presentación temática de los resultados se identificaron dos grandes narrativas: la Autotrascendencia social y las Prácticas sostenibles, que dan cuenta de un proceso por el cual la persona se posiciona en una consciencia crítica de interconexión y pensamiento sistémico con los otros, para luego ejecutar y diseminar prácticas emprendedoras responsables en beneficio de la sociedad y el mundo. Los resultados brindan insumos para considerar posibles programas de formación sobre sostenibilidad en diferentes etapas de la educación de las personas.
\end{abstract}

Palabras claves: Emprendimiento, narrativas, buenas prácticas, desarrollo sostenible.

\begin{abstract}
Appreciation for sustainable practices (ASP) is an attitudinal psychological dimension that current literature defines as a relevant component of social entrepreneurial behavior. The present study seeks to understand how this dimension is developed and it's consistent in the life narratives of 10 leaders of social enterprises in Lima, in order to state a construct that, although conceptually defined, has not been addressed from the personal experiences that make it possible. Through a qualitative methodology of narrative approach, and under a thematic presentation of the results, two major narratives were identified: Social self-transcendence and Sustainable practices. Both account for a process by which a person develops a critical awareness of interconnection and systemic thinking, to further execute and disseminate responsible entrepreneurial practices for the benefit of society and the world. The results provide inputs to consider possible training programs on sustainability in different stages of people's education.
\end{abstract}

Keywords: Entrepreneurship, narratives, best practices, sustainable development. 


\section{Introducción}

El emprendimiento social es un fenómeno que ha cobrado presencia mundial en la última década del siglo $X X I$, particularmente en las llamadas economías emergentes (Farber, Caballero, Prialé \& Fuchs, 2015; Mair \& Marti, 2006; Nicholls, 2006). Su objetivo central es promover el cambio social con la inclusión de grupos invisibilizados en el mercado tradicional (Caballero, Fusch, Prialé \& Nga, 2014; Rahdari, Sepasi \& Moradi, 2016; Zahra, Gedajlovic, Neubaum \& Shulman, 2009), atendiendo a necesidades sociales y/o ambientales que no son resueltas ni por el Estado, ni por las empresas convencionales (González, 2010; Guzmán \& Trujillo, 2008; İrengün \& Arıkboğa, 2015; Rodríguez, 2009; Verissimo, 2008).

La literatura aún no ofrece un concepto consensuado sobre el emprendimiento social. Sin embargo, sí parece existir acuerdo sobre algunas de sus características, entre las que destacan la innovación de sus modelos de negocio, la preocupación por la producción de tejido y capital social (Alter, 2003; Austin, Stevenson \& Wei-Skiller, 2006; González, 2010) y la capacidad de sus modelos para generar recursos económicos propios (Abu-Saifan, 2012; Bornstein \& Davis, 2010; Braga, J., Proença, T., \& Ferreira, M., 2014; İrengün \& Arıkboğa, 2015; Mair \& Martí, 2006; Rahdari et al., 2016; Zahra et al., 2009).

En términos generales, el emprendimiento social es un fenómeno socioeconómico orientado a la creación de valor social, que soluciona necesidades sociales y ambientales desatendidas, por medio del uso de estrategias de mercado autosostenibles (Austin et al., 2006; Mair \& Martí, 2006). Si bien un emprendimiento social necesita ser rentable e incrementar sus beneficios a lo largo del tiempo, esto solo tiene sentido al existir un propósito social que tendrá mayor impacto conforme la iniciativa logre un mejor desempeño financiero. Por ello, adopta una noción de sostenibilidad que integra beneficios económicos con una perspectiva social de largo plazo que los trasciende, calculando sus impactos a nivel social y ambiental (Ciegis, Ramanauskiene \& Martinkus 2009; Shepherd \& Patzeltz, 2011).

En contraste, el objetivo del emprendimiento llamado comercial, o tradicional, está más orientado a la asunción de riesgos en el mercado para generar mayor rentabilidad para la empresa y atender las expectativas de sus accionistas, pudiendo ello ser opuesto a la idea integral de sostenibilidad (Ciegis, et al., 2009). De allí que, desde diferentes disciplinas, se estudie el fenómeno del emprendimiento social, distanciándolo del comercial (Mair \& Marti, 2006; Nicholls, 2006).

Dado que el fenómeno emprendedor es concebido e implementado por individuos (Bornstein \& Davis, 2010), cabe preguntarse por las características psicológicas de las personas que gestan y hacen posible su existencia y sostenibilidad. De allí que la figura del emprendedor social, ofrezca un campo de estudio de particular interés, en tanto esta persona sería gestor/a y líder de la organización (Bikse, Rivza \& Riemere 2015; Estrin, Mickiewicz \& Stephan, 2016; Ovais \& Li, 2016).

Estudios sobre las características psicológicas de los emprendedores sociales señalan que, si bien comparten con los emprendedores comerciales atributos cognitivos como un estado de innovación permanente, la toma de riesgos calculados (Austin et al., 2006; Guzmán \& Trujillo, 2008; İrengün \&
Arıkboğa, 2015; Nicholls, 2006) y un alto compromiso con una tarea que implica planeación y ejecución (Rodríguez, 2009), los emprendedores sociales tienen mayor sensibilidad para identificar necesidades sociales por atender (Nga \& Shamuganathan, 2010; Seelos \& Mair 2005).

Los emprendedores sociales son capaces de identificar oportunidades de cambio social, porque tienen una visión más integral, empática y sensible sobre las necesidades tangibles y emocionales de los otros y sobre el impacto de sus negocios (Caballero et al., 2014; Guzmán \& Trujillo, 2008; Mair \& Martí, 2006; Rodríguez, 2009; Zahra et al., 2009). Por lo tanto, tienen una mayor consciencia de la noción de sostenibilidad, en tanto se sienten miembros de un sistema interconectado en el que las acciones propias y de cada una de las partes involucradas, inevitablemente afectan a las otras (Savitz \& Weber, 2006, 2014; Ovais \& Li, 2016).

Esta característica, de acuerdo a Nga y Shamuganathan (2010), es una dimensión fundamental en el comportamiento del emprendedor social denominada Aprecio por prácticas sostenibles (APS; Caballero et al., 2014). Desde una perspectiva psicosocial, la literatura señala que el APS nace de una motivación por resolver problemas socioambientales, con la convicción de que las propias acciones, ejercidas de manera equilibrada y respetuosa (Caballero et al., 2014; Kneiding \& Tracey, 2009; Nga \& Shamuganathan, 2010; Zhang \& Swanson, 2014), pueden mejorar y modificar el sistema circundante (Abu-Saifan, 2012; İrengün \& Arıkboğa, 2015; Kuckertz \& Wagner, 2010; Leisinger, 2007; Mair \& Martí, 2006).

Es así que, según plantean varios estudios en esa línea, el emprendedor social poseería un mayor estado de juicio y conciencia moral sobre el funcionamiento de la sociedad (Leisinger, 2007; Nga \& Shamuganathan, 2010) que lo conduciría a buscar el bien común, comprendiendo que tanto individuos como organizaciones conforman un mismo ecosistema interrelacionado de cuya construcción son corresponsables (İrengün \& Arıkboğa, 2015; Savitz \& Weber, 2006; Ovais \& $\mathrm{Li}, 2016)$. El objetivo de este emprendedor es generar nuevos equilibrios más estables y justos, que rompan con hegemonías excluyentes en el sistema socioeconómico imperante, tejiendo redes sociales y cooperativas que favorezcan la autosuficiencia y el autoempoderamiento (Austin et al., 2006; Verissimo, 2008).

Algunos estudios en Latinoamérica plantean que, dadas las brechas sociales de la región y la menor oferta de empleos en organizaciones de la sociedad civil (producto de la reducción de fondos de cooperación internacional), están ingresando a la actividad empresarial personas impregnadas con valores vinculados a la benevolencia, pero orientadas a la sostenibilidad y soporte financiero (Caballero et al., 2014; García \& Valencia, 2009; Moriano, 2001). Esto tendría mucho sentido en una región caracterizada por grandes asimetrías económicas, altos niveles de ausencia estatal, y una precaria seguridad social, donde la iniciativa ciudadana y privada se convierte en un camino viable para atender problemáticas sociales y ambientales (Alter, 2003).

En el Perú, una investigación realizada por Caballero et al. (2014) con 70 emprendedores sociales locales, buscó establecer relaciones entre rasgos de personalidad y dimensiones del emprendimiento social, entre ellas la del Aprecio por Prác- 
ticas Sostenibles (APS). El APS correlacionó positivamente con el rasgo de afabilidad (cooperación, empatía y cordialidad), evidenciando que la sostenibilidad está vinculada a una característica de la personalidad, que no ha sido identificada como destacable en la literatura sobre rasgos psicológicos del emprendedor comercial (Caballero et al., 2014; Zhao \& Seibert, 2006). Por su parte, Vera y Prialé (2017) en un estudio cualitativo, señalan que en emprendedores sociales de Lima, el $A P S$ es una dimensión central para entender las acciones que hacen posible la existencia y supervivencia de sus organizaciones. En efecto, desde el APS el emprendedor analiza y procesa experiencias de vida complejas que potencian la sensibilidad social y la necesidad de acción, valorando de manera sistémica las consecuencias de los propios actos y decisiones en el entorno.

Este resultado prioriza el APS como fundador de la visión social, aspecto que en otros estudios se ha señalado como inicial al surgimiento de otras dimensiones de la acción emprendedora social (İrengün \& Arıkboğa, 2015; Ovais \& Li, 2016; Waddock \& Steckler, 2016). Analizar en profundidad la manera en que se concibe y se gesta el APS, así como sus funciones psicológicas en los individuos, posibilitaría identificar las condiciones para promover un emprendimiento con mayor impacto social.

El presente estudio profundiza en los hallazgos de los estudios locales mencionados, y tiene como objetivo comprender la manera en que el APS se gesta y hace consciente en las narrativas de vida de emprendedores sociales en Lima. Se analiza también cómo esta conciencia de sostenibilidad se articula con la decisión de hacerse empresario social y con ciertas características psicológicas autoreportadas por los emprendedores en la narrativa de sus trayectorias.

\section{Participantes \\ Método}

En el estudio participaron 10 emprendedores sociales residentes en Lima Metropolitana, quienes se auto-identifican como tales y que son fundadores o miembros del equipo fundador de empresas sociales ganadoras de premios y fondos concursables, otorgados por organismos locales e internacionales. La selección de los participantes se realizó con un muestreo intencional de caso típico con máxima variación (Surin, 2011), siendo el criterio de caso típico que todos son emprendimientos sociales reconocidos localmente y, la máxima variación, que se trabajó con rubros muy diferentes, considerando encontrar narrativas comunes pese a la diferencia de intereses y de modos de acción en cada emprendedor. En el estudio participaron 5 mujeres y 5 hombres emprendedores sociales, cuyas edades oscilan entre los 25 y 50 años de edad, siendo la media de 33 años. La antigüedad de sus emprendimientos comprende entre los 2 y los 15 años, siendo la media de 4.3 años. Por otro lado, todos los emprendimientos tienen menos de 10 integrantes y clasifican en la categoría genérica de microempresas, mientras que el total de participantes tiene estudios a nivel de educación superior y sus niveles socioeconómicos oscilan entre medio $(n=8)$ y medio alto $(n=2)$. Cabe señalar que todos los participantes han brindado las entrevistas de manera voluntaria, previa aceptación de un consentimiento informado verbal. Los nombres de los participantes y las empresas han sido cambiados con el fin de mantener el principio de confidencialidad, y no se hará mención de los rubros específicos de cada empresa bajo el mismo principio. Por esta razón, en la Tabla 1 se agrupan los participantes en tres grandes rubros genéricos de sus emprendimientos.

Tabla 1. Información de los participantes

\begin{tabular}{|c|c|c|c|c|}
\hline Nombre & Edad & NSE & $\begin{array}{l}\text { Antigüedad } \\
\text { del empren- } \\
\text { dimiento }\end{array}$ & Rubro General \\
\hline Luisa & 25 & $\begin{array}{l}\text { medio } \\
\text { alto }\end{array}$ & 2 años & \multirow{5}{*}{$\begin{array}{l}\text { Cuidado } \\
\text { medioambienta }\end{array}$} \\
\hline Liliana & 26 & medio & 2 años & \\
\hline Natalia & 37 & medio & 3 años & \\
\hline Sebastián & 36 & medio & 6 años & \\
\hline José & 50 & medio & 15 años & \\
\hline Martha & 30 & medio & 2 años & \multirow{3}{*}{$\begin{array}{l}\text { Cooperativismo } \\
\text { y empleabilidad } \\
\text { inclusiva }\end{array}$} \\
\hline Darío & 31 & medio & 3 años & \\
\hline Álvaro & 41 & medio & 6 años & \\
\hline Renato & 28 & $\begin{array}{l}\text { medio } \\
\text { alto }\end{array}$ & 2 años & $\begin{array}{l}\text { Acceso a } \\
\text { servicios }\end{array}$ \\
\hline Luz & 30 & medio & 2 años & $\begin{array}{l}\text { básicos y } \\
\text { saneamiento }\end{array}$ \\
\hline
\end{tabular}

Instrumento

Se realizó una entrevista narrativa abierta en profundidad (Creswell, 2013), que partió de tres premisas: "Cuénteme cómo llegó a hacerse emprendedor social", "Para usted, ¿qué significa la sostenibilidad?" y “¿Cómo la aplica en su vida y en su emprendimiento?". Se buscó indagar sobre la forma en la que los emprendedores participantes relatan, desde sus experiencias de vida, el proceso por el que llegaron a concebir y liderar su emprendimiento social, consolidando una noción propia de sostenibilidad y un APS. Dado que se trata de una entrevista narrativa, la misma se valida en el proceso de aplicación en conjunto con el participante, quien guía con sus propias ideas, valores y experiencias la narración, siendo el trabajo del investigador el de escuchar activamente y facilitar por medio de repreguntas que el participante profundice en los detalles de las vivencias para reconstruir su propia narrativa (Schwartz \& Jacobs, 2006).

\section{Procedimiento}

Para lograr el objetivo de la investigación, se realizó un estudio cualitativo con un diseño de enfoque narrativo, el cual busca recopilar las historias de los participantes desde sus propias experiencias, enfocándose tanto en las personas como en el contexto, para realizar la interpretación organizada de una serie de eventos desde los que se puedan inferir relaciones de causalidad. La construcción de la narrativa se dio en la interacción empática entre el investigador y los participantes (Creswell, 2013).

Para conformar el grupo de participantes, en primer lugar, se elaboró una base de datos con información de emprendimientos sociales en Lima que hubieran sido ganadores de reconocimientos y fondos en los últimos 5 años, de allí se armó una base inicial con los 25 emprendimientos que además habían tenido más divulgación mediática. Entre ellos, se preseleccionaron 16 emprendimientos que representaran diferentes campos de acción social y/o ambiental. Luego se procedió a 
contactar a los emprendedores y finalmente, se trabajó con aquellos que tuvieron el interés y disponibilidad de tiempo para participar en la investigación. Las entrevistas tuvieron una duración promedio de 120 minutos y se programaron de común acuerdo con los emprendedores en sus lugares de trabajo, tras la aceptación del consentimiento informado que garantizaba la confidencialidad y brindaba los objetivos y características del estudio.

\section{Análisis de información}

Las entrevistas fueron transcritas y analizadas utilizando el programa ATLAS.ti v. 7.5. Para el análisis de las entrevistas, se empleó la metodología de análisis narrativo (Creswell, 2013), que consiste en organizar de manera cronológica la información brindada por cada participante, identificando las historias o situaciones claves donde se explicitan desenlaces significativos en la narración; en este caso, todos aquellos relacionados con la configuración del APS y la decisión de hacerse emprendedor social. Si bien cada narrativa es única en sí misma a nivel individual, el posterior análisis buscó agrupar aquellas situaciones claves que resultaran relativamente comunes a todos los participantes, para con ellas construir categorías generales que, desde una presentación temática (Braun \& Clarke, 2006), permitan comprender la consolidación del APS en el proceso emprendedor.

\section{Resultados}

El análisis integró las experiencias claves de los participantes, asociadas a la manera en que se configura una noción de sostenibilidad, en dos grandes narrativas. En primer lugar, la narrativa de Autotrascendencia social relata, en dos subnarrativas: Privilegios y responsabilidades e Integración trascendente con el entorno, los procesos que acompañan una toma de consciencia trascendente, determinante en la decisión de hacerse emprendedor. Ello da lugar a la configuración de la segunda narrativa sobre Prácticas sostenibles, en la que se reportan las acciones concretas que ejecutan, cotidianamente como emprendedores, para materializar su posicionamiento autotrascendente, presentando dos tipos de prácticas: Coherencia entre procesos e impactos y Posibilidad de inspirar y transformar. A continuación, se detallan y discuten los resultados en cada narrativa.

\section{Autotrascendencia social}

Esta narrativa está vinculada a los procesos de socialización significativos (familiares, educativos, experienciales, etc.) que fueron dando lugar a desarrollar la sensibilidad social de los participantes y gestaron su aprecio por la sostenibilidad y su intención emprendedora. En todas las narrativas de los participantes es posible encontrar articulaciones complejas y conscientemente analizadas por ellos mismos, entre sus experiencias de vida y los efectos sobre sus decisiones emprendedoras. Desde dichas articulaciones se evidencia el enriquecimiento en ellos de una mirada benevolente y universalista, libre de ansiedades y con características auto-expansivas, sobre las cuales asumen la búsqueda de un sentido auto-trascendente de la propia vida (Schwartz, 1992; García \& Valencia, 2009).

Las narrativas de todos los participantes asociados a la autotrascendencia social presentan de manera consistente una serie de explicaciones sobre cómo, a lo largo de la historia personal, desde la crianza inicial, hasta procesos educativos y de socialización más tardíos, se ha dado una toma de conciencia del nivel de responsabilidad y articulación que se tiene como persona en un sistema más amplio e interdependiente.

\section{Privilegios y responsabilidades}

En esta subdimensión de la autotrascendencia social, los participantes, independientemente de la condición socioeconómica en la que se hayan criado, se reconocen como personas privilegiadas en un contexto social como el peruano, que no siempre es justo y en el que ellos han contado con suerte. La consciencia de la buena suerte en sus vidas es una constante en las narrativas de los participantes. El saberse aventajados en aspectos económicos, educativos y particularmente, en haber tenido una socialización respetuosa, considerada y empática, es leído por ellos como un afortunado privilegio, que de ninguna manera asumen como un derecho o mérito logrado per se, pues reconocen que han contado con la suerte que la mayoría de sus compatriotas no ha tenido.

"Yo creo que como persona he recibido muchas cosas en la vida y eso es por suerte, o sea, uno nace donde nace por suerte, mi familia me llenó de afecto (...). Mis abuelos tenían un alma, un tema de servicio. $Y$ yo creo que desde chica ese tema lo pasaron a mis papás y mis papás a nosotros. $Y$ temas como viajar por Perú para conocerlo, ver que todo lo que tú has recibido no todos lo han recibido, representa una gran responsabilidad para uno. Entonces yo creo que, desde chica, yo creo que es un tema de valores y lo vas recibiendo en tu casa, uno tiene la responsabilidad de devolver lo que ha recibido" (Luisa, comunicación personal, octubre 2016)

"Perú es un país pobre y jodido y yo tuve un montón de suerte. Bueno, lo mínimo que puedo hacer es tratar de hacer algo para que no sea tan jodido y otra gente pueda tener las mismas oportunidades que yo he tenido, ¿no?" (Martha, comunicación personal, octubre 2016)

Esta conciencia de buena suerte, según señala la literatura, conlleva a que las personas sean más optimistas y usen estrategias de afrontamiento más efectivas frente a retos o situaciones potencialmente difíciles (Parnell \& Dent, 2009; Whittington, 2016), asumiendo comportamientos adaptativos y racionales que aseguren el éxito de sus iniciativas de manera más persistente y positiva (Liu \& De Rond, 2016). Esto convoca un sentido de deber moral que busca ayudar para equilibrar de manera sostenible las propias ventajas con respecto a aquellos que están del otro lado de la balanza (Mancilla, 2015; Pogge, 2008; Stoll, 2015). El reconocimiento de estos privilegios, como lo evidencian las citas, revierte en asumir una posición responsable frente al entorno y en una necesidad de devolver y compensar al mundo por las ventajas que se han tenido respecto a otros seres humanos.

"Y también tenía la convicción de que yo quiero; yo siento que es mi deber o pasión ayudar. O sea, era como mi deber, como un "tengo que". No es justo que haga algo solamente que a mí me divierte o me sirve, sino tengo que hacer (...). Era algo, era de verdad, como un deber con todo lo que se me había dado en la vida" (Luz, comunicación personal, octubre 2016). 
Esta idea de retribuir desde la buena fortuna propia, siendo responsable por mejorar las condiciones de otros, hace parte de la configuración de una noción de sostenibilidad que revierte el actuar en beneficio de la sociedad, sumando un retorno económico (Stoll, 2015). La consciencia de los propios privilegios, constituye un antecedente importante en la búsqueda de equilibrios sobre las desigualdades sociales para erradicarlas a través de estrategias innovadoras (Leisinger, 2007; Pralahad, 2010; Pogge, 2008).

\section{Integración trascendente con el entorno}

El reconocimiento de privilegios y responsabilidades, presentado en la dimensión anterior, se complementa con la coherencia de los participantes para dar cuenta, en sus narrativas, de aquellas experiencias, particularmente formativas, que les han permitido reconocerse como parte un sistema retroalimentado de manera más amplia, sintiéndose integrados a un equilibrio mayor en el que deben aportar activa y responsablemente, siendo el emprendimiento un camino de interés.

"Debe de haber sido desde el cole, porque siempre hubo el tema de las misiones a Ayacucho, el tema de las misiones a la selva que salían en secundaria, el tema, estuve buen tiempo en los scouts también en el cole, eso me llenó de consciencia y sobre todo de un deseo de actuar, de emprender para lograr soluciones" (Sebastián, comunicación personal, octubre 2016)

"Creo que mi misma crianza o mí misma experiencia de vida (...). Yo fui a un colegio que promueve mucho los valores y Derechos Humanos en el sentido de que era un colegio internacional y he crecido con niños de todos los lados del mundo (...). Eso te enseña mucha tolerancia de culturas (...), lo social es más por mi familia, por mi papá que trabajó más de 30 años en una ONG internacional. Cuando yo era niña, él trabajaba en micro-emprendimiento en regiones vulnerables, en mujeres mayormente de esa zona. Yo me acuerdo que teníamos juegos de mesa que se usaban para talleres en la casa y decía vamos a jugar al juego del emprendimiento" (Liliana, comunicación personal, octubre 2016)

En las narrativas se encuentra, de manera reiterada, la noción de relación y retroalimentación continua entre los propios actos y sus efectos en las demás personas y el ambiente, producto de la posibilidad de entrar en contacto con contextos, prácticas y culturas diferentes a la propia. De igual forma, en la oportunidad de tener acceso a información enriquecida y compleja sobre algún tema de interés, para desarrollar alternativas de acción sobre el mismo con un pensamiento crítico.

"Mi padre fue un ingeniero agrónomo que estudió en Chile, en la etapa del fin de la agricultura autosostenible y natural, previo a esta ola de la llamada revolución verde, la maquinización, los fertilizantes, etc., la agricultura que ha malogrado la vida de la gente en términos de alimentación. Entonces mi padre tenía para sus estudios literatura de esa agricultura y tenía un montón de revistas, había una revista argentina que se llama "mi chacra" y una chilena "la hacienda", y bueno una colección enor-

Summa Psicológica UST (CC - BY - 3.0)

ISSN: 0718-0446 / ISSNe: 0719-448x

http://summapsicologica.cl/ me en la casa, yo he gozado mucho de eso siendo estudiante" (José, comunicación personal, octubre 2016)

Como se puede ver en las citas, la participación activa en grupos educativos, así como el contacto con diferentes escenarios de la realidad, sumado a un reconocimiento histórico y contextual claro de sus circunstancias formativas, es una constante en las narrativas de los emprendedores sociales participantes. El viajar, salir a campo y tener contacto con otras experiencias, haría parte del proceso para configurar un sentido de integración trascendente con el entorno, que consiste en reconocer que no se está solo, que existen muchos modos de realidad y que se puede aportar a las mismas integrando saberes y prácticas (Braga et al., 2014).

"Desde que estaba en el colegio, yo participaba en los boyscout, ahí hay un tema de conocimiento fuera de Lima de campo, porque tuve la oportunidad de estar unos años antes del terrorismo, donde sí podíamos hacer, pues, salidas a campo y todo eso, eso marcó una etapa en mi vida, después cuando vino toda esta época, yo participaba mucho de asociaciones juveniles" (Álvaro, comunicación personal, octubre 2016)

Esto posibilita un pensamiento descentrado de sí mismo, más holístico y respetuoso de la alteridad, lo que introduce un fuerte sentido de responsabilidad y conciencia por las consecuencias que las decisiones personales y empresariales pueden traer a corto, mediano y largo plazo. Las citas ejemplifican también una característica presente en todos los participantes, que es la conciencia explícita de estar en relación y retroalimentación continua con el entorno. Ello puede asociarse con las particularidades del pensamiento sistémico, el cual explica una aproximación hacia una conciencia crítica y social, que busca el desarrollo del potencial humano mediante el trabajo y la organización (Martínez, 2013).

El pensamiento sistémico postula un contexto complejo, en el que actuar de manera sostenible, requiere de concebir el contexto como un sistema interconectado, donde la totalidad de sus elementos y/o actores se influyen entre sí mediante interacciones recursivas y relaciones dinámicas (Lampis, 2013). En el discurso de los entrevistados, el pensamiento sistémico se vislumbra en la sensación de estar en conexión y ser parte de una sociedad que es susceptible de ser transformada. Esta visión holística parte de reconocer que las relaciones o interacciones dentro de los elementos del contexto -o sistema- son más importantes que los elementos en sí mismos (Liévano \& Londoño, 2012; Martínez, 2013).

"Vivimos con mi familia fuera del Perú muchos años, solo veníamos en el verano unas semanas a visitar a mi abuela. Yo, desde que recuerdo, siempre me preguntaba por qué las cosas eran tan diferentes, cómo podía yo pertenecer (...) pero, recuerdo siempre haber sentido, quizás que sí, que yo era parte de todo, de esta sociedad y de la otra, no me podía sentir ajena. Veía los techos de esteras y me dolía (...). Siempre he sentido que todo está en conexión, que soy responsable, que no puedo hacer solo lo que yo quiero, que cuando algo no está conectado debes hacer el esfuerzo para que lo esté, somos parte de la realidad que creamos" (Luz, comunicación personal, octubre 2016) 
La teoría señala que la conciencia o visión social en los emprendedores sociales podría fundamentarse en la vivencia de experiencias contrastantes (Ovais \& Li 2016; Waddock \& Steckler 2016), lo cual es claro en los participantes, pero como paso previo se alimenta de la noción de sostenibilidad. Como lo señala el estudio de Prialé y Vera (2017), que analiza cinco dimensiones del emprendedor social, la noción de sostenibilidad -o Aprecio por Prácticas Sostenibles- es una categoría central y fundamental en los emprendedores sociales, pues articula y permite dar sustento cognitivo a la visión social. La sostenibilidad y su sentido de interconexión nace de una historia personal compleja, atravesada por experiencias enriquecedoras en contextos diversos, que permite valorar de manera sistémica las consecuencias de los propios actos y decisiones, sirviendo como base para un posterior sentido de visión social (Lampis, 2013; Prialé \& Vera, 2017). Este posicionamiento sería reflejo de valores universalistas en los que los emprendedores sociales se conciben como ciudadanos del mundo y corresponsables de las realidades en las que hay alguna necesidad social o ambiental urgente de atender.

\section{Prácticas sostenibles}

En esta narrativa se da cuenta de todas las acciones concretas y tangibles que han realizado los emprendedores a lo largo de su vida para mantener una coherencia con su sentido de corresponsabilidad con el entorno, siendo conscientes de los impactos reales que sus actividades representan. Esto implica imaginar los efectos proyectados, tanto de acciones mínimas y cotidianas como de acciones macrosociales, que puedan cambiar por completo el sistema productivo y social inspirando a otros.

Los participantes reiteran la noción de relación y retroalimentación continua entre los propios actos y sus efectos en las demás personas y el ambiente. Esto introduce un fuerte sentido de responsabilidad y conciencia por las consecuencias que las decisiones personales y empresariales pueden traer a corto, mediano y largo plazo, requiriendo buscar un balance integrador entre lo económico y lo social-ambiental (Hawken 1993; İrengün \& Arıkboğa 2015; Leisinger 2007).

Un elemento importante en esta narrativa de los emprendedores, es el sentido de autosuficiencia (Gumus, 2014) y autoeficacia (Drnovsek, Wincent \& Cardon, 2010), que les permite ejercer su labor y sentir que son capaces de llevar a cabo su resultado social, pese a las dificultades que puedan presentarse en el entorno. La autosuficiencia es referida a la autoevaluación de las propias capacidades para demostrar habilidades y un desempeño exitoso (Bandura, 1997). En el contexto del emprendimiento, es clave no solo para tener éxito en el mismo, sino que la autosuficiencia se ve retroalimentada e incrementada con la percepción del éxito y soporte organizacional (Gumus, 2014), lo que estaría en vínculo con la consciencia optimista de privilegios analizada en la narrativa anterior, en la búsqueda de objetivos tangibles y sostenibles para el emprendimiento (Drnovsek, Wincent \& Cardon, 2010).

\section{Coherencia entre procesos e impactos.}

Todas las narrativas de los participantes dan cuenta de una reflexividad sobre los efectos que las decisiones personales y empresariales conllevan en las personas y el entorno. Se trata de un posicionamiento orientado a la sostenibilidad, en el que se piensa no solo en los resultados finales esperados sino ante todo, en los impactos que el proceso mismo conlleva, considerando que en cada momento se generen beneficios para las personas y el entorno (Bikse, Rivza \& Riemere 2015; İrengün \& Arıkboğa, 2015; Zhang \& Swanson, 2014):

"El cooperativismo me inspiró, encontré que había los medios económicos, los medios académicos y sociales para poder ayudar a otras personas. Nuestro objetivo era igual, bajo la misma filosofía, que el impacto lo podamos medir, sea razonable, haya mayor inclusión y acceso" (Álvaro, comunicación personal, octubre 2016)

"Ejercemos la habilidad para el cambio, que también se le llama como gestión de la acción que es la habilidad para poder gestionar un cambio, para poder gestionar una acción y tener un impacto en tu entorno" (Darío, comunicación personal, octubre 2016)

La teoría señala que para el logro de la sostenibilidad, la ejecución de prácticas y modelos económicos innovadores es importante (Mair \& Martí, 2006), lo que implica, como lo ejemplifican las anteriores citas, estar muy conscientes de los recursos con los que se cuenta, así como ser rigurosos en el seguimiento y monitoreo de las acciones que se llevan a cabo para el logro de los objetivos del emprendimiento (İrengün \& Arıkboğa, 2015; Mair \& Martí, 2006; Mair \& Schoen, 2007; Nicholls, 2006). De igual manera, supone orientarse al cambio y mejoramiento de la sociedad, buscando incluso expandirse y escalar sus impactos sociales con una mirada sistémica y con diversos niveles de alcance:

\begin{abstract}
"Me encantaría que se convierta en un proyecto regional, sólido así de largo alcance, que realmente logremos una transformación no solo a nivel individual en las chicas que pasan en el programa y en todos los que somos parte del programa, pero también a nivel sistema, ¿no? Como que, que realmente logremos construir países donde haya mayor igualdad de oportunidades, donde aprovechemos el sector de tecnología para crear nuevas formas de aprender, de enseñar y de trabajar, que den más oportunidades a quienes tienen las ganas de sacarles el provecho" (Martha, comunicación personal, octubre 2016)
\end{abstract}

Para los participantes, debe haber una coherencia entre metas y procesos, lo que implica el querer hacer las cosas bien. Desde las relaciones mismas que se generan en sus empresas, con sus colaboradores, hasta los alcances con sus destinatarios, se percibe un sentido de horizontalidad y cuidado por cada una de las acciones y sus efectos, y sobretodo un cuidado por el otro, buscando siempre un balance que permita soluciones innovadoras y equilibradas con las necesidades del entorno. Lo que estaría en consonancia con las correlaciones identificadas entre el APS y la afabilidad por los otros (Caballero et al., 2014). Estas acciones conllevan un sentimiento de bienestar y satisfacción personal.

"Ética, coherencia, transparencia eso es bien importante, porque te genera confianza entre todos, porque trabajamos juntos y somos solidarios, la solidaridad adentro de la empresa es muy importante 
a nivel de las orientaciones del desarrollo. Te das cuenta que no tienes que centrarte en ti (...), sino en el prójimo. Entonces te aproximas, reconoces la necesidad y aportas para cubrirla (...), buscas también que las personas se hagan conscientes, tocas vidas, cambias el pensamiento y cambian las acciones. Me siento muy satisfecha de participar en todo esto" (Natalia, comunicación personal, octubre 2016)

Se percibe, entonces, un claro posicionamiento axiológico de orientación universalista y benevolente donde la coherencia e integridad son centrales para mantener un posicionamiento moral consistente (Schwartz et al., 2009). Consecuentemente, ello resulta en un beneficio mutuo en el que no solo el contexto es recompensado con prácticas económicas sostenibles, sino que el propio emprendedor se ve recompensado en su bienestar psicológico que le provee de sentido (SoosaiNathan, 2015). En los participantes el APS integra aspectos emocionales con una alta capacidad de razonamiento lógico y práctico, orientándose a la consecución de resultados sostenibles, efectivos e idealmente medibles que permiten planificar y tomar decisiones (Prialé \& Vera, 2017).

Posibilidad de inspirar y transformar.

Esta última narrativa está asociada a la manera en que los participantes conciben acciones en sus empresas desde el deber moral de posicionarse como líderes inspiradores, capaces de transformar estados desequilibrados de la realidad (Helmsing, 2015; Seelos \& Mair 2005), brindando a sus destinatarios y al público en general la posibilidad de participar y hacerse conscientes de los impactos de sus propias acciones (Kneiding \& Tracey, 2009; Nga \& Shamuganathan, 2010; Ovais \& Li 2016; Zhang \& Swanson, 2014). Esta narrativa es producto de la anterior, pues solo se logra inspirar cuando se es coherente y consciente de los procesos e impactos que posibilitan el cambio de un estado de la realidad. Esto conlleva a que otros también tomen conciencia de estos impactos y piensen sus acciones de manera sostenible y sistémica.

"Destinamos el $100 \%$ de las utilidades al proyecto social. El consumidor tiene ahora la opción de ir a un punto de venta y comprar nuestro producto al mismo precio de otros, con la misma calidad, pero le estamos dando el sentido de posibilidad: "oye, tú, compra" y estás cambiando vidas. Con tu compra le vas a dar por lo menos ocho días de agua limpia a una persona en condiciones de pobreza extrema. Entonces generamos el sentido de posibilidad, sentido de conciencia de que hay ocho millones de peruanos que no tienen agua, entonces a través de publicidad, redes sociales que es nuestro fuerte, comunicaciones, entrevistas, damos a conocer esta realidad, para que luego esas personas que ya han tenido el sentido de posibilidad puedan tomar decisiones, hacer acciones indirectas o directas hacia la causa en su círculo social" (Renato, comunicación personal, octubre 2016)

Entonces, se piensa en inspirar, concientizar y en alcances mayores que implican divulgar y visibilizar los proyectos, mostrando que se pueden lograr resultados positivos y experiencias exitosas haciendo las empresas de otra manera. Esta perspectiva tiene un claro componente de pensamiento com- plejo, porque requiere de movilizar relaciones y acciones en un sistema más amplio, para dar escala y proyección a las metas sociales y/o ambientales que promueven (Lampis, 2013; Liévano \& Londoño, 2012; Martínez, 2013; Roitman, 2004). Adoptar una idea de interrelación con el contexto y sociedad, condiciona el deseo y necesidad expansiva que busca inspirar para transformar. Se trata de hacerlo con el propio ejemplo y la propia coherencia.

"Nuestro emprendimiento ha sido esa experiencia que cambia, parte del beneficio que genera es que buscamos estar centrados en la persona, en la familia peruana, en el acceso a oportunidades. $Y$ esos son temas a los que queremos darles alto alcance. Por eso hoy lo hago en una escala más grande, buscando participar en eventos internacionales, divulgar la experiencia, hacer contactos, participar en redes y programas que le den visibilidad a nuestro proyecto (...) pero no por nosotras, sino por inspirar a otros a ver que sí se puede, que podemos hacerlo, que unidos somos capaces porque nadie dijo que las empresas tienen que ser como siempre han sido. Podemos dar ejemplo de eso" (Luisa, comunicación personal, octubre 2016)

Y todo ello, como lo reiteran los participantes, se da con miras a hacer las cosas de otra manera, mostrar que es posible y que se puede crear un entorno empresarial y productivo justo, inclusivo y responsable sobre sus acciones. En esta narrativa confluye y se reedifica la idea del aprecio por prácticas sostenibles, entendiendo la sostenibilidad en una perspectiva conjunta, colaborativa y sensible para dar oportunidades a los otros.

\begin{abstract}
"Tratamos de generar propósito, ¿no? La posibilidad de motivar a la gente para unirse a una causa que nos trasciende. $Y$ especialmente, el crear espacios donde las personas podemos aprender y donde se generan espacios de interacción social positiva, de acciones colectivas, escalables, de gobernanza descentralizada, de comunicación afectiva, especialmente en el tema ambiental" (Darío, comunicación personal, octubre 2016)
\end{abstract}

Así, las Prácticas Sostenibles no solo constituyen una herramienta para la supervivencia del emprendimiento, sino que se confluyen como un posicionamiento personal que influye en otros con conciencia crítica de las consecuencias de los propios actos sostenibles e interconectados (Lampis, 2013). Se puede dar cuenta de una visión "ecológica" o "ecosistémica", que más allá de lo ambientalista se piensa como interactuante y co-constructora de su entorno. Es así que, en los emprendedores participantes, su visión social es emotiva, optimista y confiada en sus posibilidades para transformar una realidad (Chell et al. 2016; Waddock \& Steckler 2016). Las dos citas a continuación resumen claramente la idea de sostenibilidad que manejan todos los participantes:

"En una industria de alto crecimiento como la tecnológica se pierde de vista la sostenibilidad a largo plazo, se debe crecer, pero hacerlo de una manera responsable, en la que le podamos dar oportunidad a personas que de otra manera nunca la tendrían. Entonces ahí está el impacto del programa: 
crecemos juntos y nos sostenemos juntos. No tendría sentido hacerlo solos" (Martha, comunicación personal, octubre 2016)

"Sostenibilidad para mí es vivir con conciencia de los impactos que nuestras decisiones generan a nivel económico, social y ambiental. Ser consciente que esas tres aristas tienen que ser consideradas y ver cuál es el impacto a corto, mediano y largo plazo de las decisiones que tomas en el día a día" (José, comunicación personal, octubre 2016)

\section{Discusiones}

El análisis de las narrativas de los participantes ha permitido profundizar en la comprensión de los acontecimientos autobiográficos, los posicionamientos personales y las acciones conscientes y voluntarias que dan contenido y forma a la configuración de la dimensión del APS, la cual, según estudios locales previos, ha sido identificada como central en las características y el accionar de los emprendedores sociales locales (Caballero et al., 2014; Prialé \& Vera, 2017).

Se puede concluir que el aprecio por prácticas sostenibles se consolida en una conciencia sistémica, que implica pensar las relaciones y efectos de las propias acciones sobre el mundo, reconociéndose como un agente corresponsable de las condiciones en que se produce la realidad, lo que en este estudio se ha representado en la narrativa de integración trascendente con el entorno. Esta búsqueda de acciones concretas transformadoras, que en el estudio son captadas en las narrativas asociadas a Prácticas sostenibles (con sus dos narrativas específicas), se reconoce como una respuesta coherente y recíproca de los participantes con su sociedad, dada la consciencia de tener una vida privilegiada que los hace responsables para tomar acciones (explicada en la narrativa de privilegios y responsabilidades).

Todos los participantes perciben haber vivido experiencias enriquecidas por el contacto con otras realidades diferentes a las propias, así como haber tenido un entorno formativo (familiar y educativo) tolerante, afectivo y consistente con una visión sistémica y corresponsable con la realidad. La identificación de estos hitos narrativos biográficos lleva a poner la mirada sobre las implicaciones que los procesos educativos tendrían en la formación de futuros emprendedores sociales responsables con la resolución de necesidades en su entorno.

Dado que los hallazgos narrativos de este estudio, no remiten solo a vivencias primarias de la infancia de los participantes, sino a una transversalidad de experiencias e información capitalizada por ellos para fundamentar su actuar, resulta relevante interpelar al mismo entorno académico-universitario (GiraldoMejía \& Vara-Horna, 2018), para analizar los procesos de educación superior y los contenidos axiológicos y reflexivos sobre los cuales se forman los futuros empresarios y profesionales para la sociedad. En asociación con esta interpelación y con la teoría revisada sobre el pensamiento sistémico vinculado a la conciencia de sostenibilidad (que indica que esta modalidad de pensamiento puede educarse y enriquecerse a lo largo de la vida) (Chappin, Bijvoet, \& Oei, 2017), sería importante entonces preguntarse por las condiciones de intervención que se pueden llevar a cabo desde entornos educativos diversos, en diferentes etapas del desarrollo, para fomentar el APS que conlleve al empoderamiento y la acción social.

Se recomienda, de acuerdo a lo analizado, considerar la toma de consciencia de las propias habilidades y recursos, la reflexión optimista y autoeficaz sobre la posibilidad de agenciarse y actuar efectivamente con la responsabilidad de revertir bienestar a otros y la capacidad de medir impactos e inspirar a todas las partes implicadas. La educación con un enfoque en sostenibilidad, resulta crucial a nivel institucional y social, por lo que es imprescindible un enfoque holístico donde se piense en la sostenibilidad no solo de la institución, sino de ideas y valores que contribuyan a una transformación genuina de un mundo en crisis (Bornstein \& Davis, 2010).

En cuanto a las limitaciones del estudio, se trata de una muestra pequeña y local, lo que no permite la generalización de los resultados a otros contextos, aunque podría hipotetizarse que hay una constante para el caso peruano pues las temáticas narrativas reportadas, a pesar de provenir de experiencias muy diversas, resultan comunes a todos los participantes. Es así que se plantea la posibilidad de avanzar hacia estudios de carácter cuantitativo que permitan confirmar, con representatividad estadística, las características de esta dimensión del aprecio por prácticas sostenibles en empresarios sociales y comerciales peruanos, y correlacionarla con otros rasgos psicológicos posiblemente asociados, como la personalidad, los valores e incluso posicionamientos ideológicos, lo que permitiría un mayor conocimiento del constructor y un mayor fundamento para pensar en programas de formación del mismo. Todo esto desde una mirada sistémica, relevante en el contexto de la economía emergente e inequitativa que caracteriza la realidad peruana y latinoamericana.

\section{Referencias}

Abu-Saifan, S. (2012). Social Entrepreneurship: Definition and Boundaries. Technology Innovation Management Review. February 2012: 22-27.

Alter, S. (2003). Social enterprise: A typology of the field contextualized in Latin America. Washington, DC: Inter-American Development Bank.

Austin, J., Stevenson, H., \& Wei-Skiller, J. (2006). Social and Commercial Entrepreneurship: Same, Different or Both? Entrepreneurship Theory and Practice, 30(1), 1-22.

Bandura, A. (1997). Self-efficacy: The Exercise of Control. New York, NY: Freeman.

Bikse, V., Rivza, B., \& Riemere, I. (2015). The Social Entrepreneur as a Promoter of Social Advancement. Procedia - Social and Behavioral Sciences, 185(13), 469-478.

Bornstein, D., \& Davis, S. (2010). Social Entrepreneurship. What everyone needs to know. New York, NY: Oxford University Press.

Braga, J., Proença, T., \& Ferreira, M. (2014). Motivations for social entrepreneurship - Evidences from Portugal. Tékhne Review of Applied Management Studies,12(1), 11-21.

Braun, V. \& Clarke, V. (2006). Using thematic analysis in psychology. Qualitative Research in Psychology, 3(2), 77-101.

Caballero, S., Fuchs, R., Prialé, M., \& Nga, J. (2014). The influence of the Big 5 personality traits on the social 
enterprise start-up intentions: A Peruvian case. Taylor's Business Review, 4(1), 87-107.

Chappin, E., Bijvoet, X., \& Oei, A. (2017). Teaching sustainability to a broad audience through an entertainment game - The effect of Catan: Oil Springs. Journal of Cleaner Production, 156(10), 556-568.

Ciegis, R., Ramanauskiene, J., \& Martinkus, B. (2009). The concept of sustainable development and its use for sustainability scenarios. Inzinerine EkonomikaEngineering Economics, 2, 28-37.

Creswell, J. (2013). Qualitative Inquiry \& Research Design: Choosing Among Five Approaches. Thousand Oaks, CA: Sage.

Drnovsek, M., Wincent, J., \& Cardon, M. (2010). Entrepreneurial self-efficacy and business start-up: developing a multi-dimensional definition. International Journal of Entrepreneurial Behaviour \& Research, 16(4), 329-348.

Estrin, S., Mickiewicz, T., \& Stephan, U. (2016). Human capital in social and commercial entrepreneurship. Journal of Business Venturing, 31(4), 449-467.

Farber, V., Caballero, S., Prialé, M.., \& Fuchs, R. (2015). Social Enterprises in Lima: Notions and Operating Models. Journal of Entrepreneurship and Innovation in Emerging Economies, 1(1), 56-78.

García, P., \& Valencia, P. (2009). Factores de éxito del proceso de puesta en marcha de la empresa: valores culturales, redes sociales y ayudas públicas. Investigaciones Europeas de Dirección y Economía de la Empresa, 15(3), 101-117.

Giraldo-Mejía, W. \& Vara-Horna, A. (2018) El emprendimiento social de base universitaria en latinoamérica ¿Están las universidades haciendo lo suficiente? Lima, Perú: Universidad de San Martín de Porres. Facultad de Ciencias Administrativas y Recursos Humanos.

González, M. (2010). Emprendimiento social. Tejidos de confianza y mercados inclusivos. Revista Javeriana. 146(765), 28-37.

Gumus, S. (2014). The Relationship between Organizational Support Perceptions and Self-Sufficiencies of Logistics Sector Employees. International Journal of Research in Business and Social Science, 3(2), 121147.

Guzmán, A., \& Trujillo, M. (2008). Emprendimiento social - Revisión de Literatura. Estudios Gerenciales, 24(109), 105-125.

Helmsing, A. (2015). Conditions for Social Entrepreneurship. The International Journal of Social Quality, 5(1), 5166.

İrengün, O., \& Arıkboğa, S. (2015). The Effect of Personality Traits on Social Entrepreneurship Intentions: A Field Research. Procedia - Social and Behavioral Sciences, 195, 1186-1195.

Kneiding, C., \& Tracey, P. (2009). Towards a performance measurement framework for community development finance institutions in the UK. Journal of Business Ethics, 86(3), 327-345.

Kuckertz, A., \& Wagner, M. (2010). The influence of sustainability orientation on entrepreneurial intentions - Investigating the role of business experience. Journal of Business Venturing, 25(5), 524-539.

Lampis, M. (2013). Del texto a la cultura. Apuntes sobre el pensamiento sistémico aplicado a los estudios cultu- rales. Revista Signa, 22, 447-462.

Leisinger, K. (2007). Corporate Philanthropy: The Top of the Pyramid. Business and Society Review, 112(3), 315342.

Liévano, F., \& Londoño, J. (2012). El pensamiento sistémico como herramienta metodológica para la resolución de problemas. Revista Soluciones de Postgrado EIA, 4(8), 43-65.

Liu, C., \& De Rond, M. (2016). Good Night, and Good Luck: Perspectives on Luck in Management Scholarship. The Academy of Management Annals, 10(1), 409451.

Mair, J., \& Martí, I. (2006). Social entrepreneurship research: A source of explanation, prediction, and delight. Journal of World Business, 41(1), 36-44.

Mair, J., \& Schoen, O. (2007). Successful social entrepreneurial business models in the context of developing economies. An explorative study. International Journal of Emerging Markets, 2(1), 54-68.

Mancilla, A. (2015). What the old right of necessity can do for the comtemporary global poor. Jorunal of Applied Philosophy, 34(5), 607-620.

Martínez, L. (2013). Una aproximación contextual y conceptual al pensamiento de sistemas. Revista Soluciones de Postgrado EIA, 6(11), 101-119.

Moriano, J., Trejo, E., \& Palací, F. (2001). El perfil psicosocial del emprendedor: un estudio desde la perspectiva de los valores. Revista de Psicología Social, 16(2), 229-242.

Nga, J., \& Shamuganathan, G. (2010). The Influence of Personality Traits and Demographic Factors on Social Entrepreneurship Start Up Intentions. Journal of Business Ethics, 95(2), 259-282.

Nicholls, A. (2006). Social Entrepreneurship: New Models of Sustainable Social Change. Oxford: Oxford University Press.

Ovais, M., \& Li, C. (2016). Personality Traits and their Effects on Social Entrepreneurship Intention. The International Journal of Business \& Management, 4(4), 222-226.

Pogge, T. (2008). World Poverty and Human Rights: Cosmopolitan Responsibilities and Reforms. Malden, MA: Polity Press.

Parnell, J., \& Dent, E. (2009). The role of luck in the strategyperformance relationship. Management Decision, 47(6), 1000-1021.

Pralahad, C. (2010). The Fortune at the Bottom of the Pyramid: Eradicating Poverty Through Profits. New Jersey: Pearson Prentice Hall.

Prialé, M. \& Vera, A. (2017). Análisis de las dimensiones teóricas del emprendimiento social desde las experiencias de empresarios sociales en Lima Metropolitana. Lima: Centro de Investigación de la Universidad Pacífico.

Rahdari, A., Sepasi, S., \& Moradi, M. (2016). Achieving sustainability through Schumpeterian social entrepreneurship: The role of social enterprises. Journal of Cleaner Production, 137(20), 347-360.

Rodríguez, A. (2009). Nuevas perspectivas para entender el emprendimiento empresarial. Pensamiento y Gestión, 26, 94-119.

Roitman, M. (2004). El Pensamiento Sistémico: Los orígenes del Social-Conformismo. México, D.F.: Siglo XXI.

Savitz, A., \& Weber, K. (2006). The Triple Bottom Line: How 
today's Best-Run Companies Are Achieving Economic, Social, and Environmental Success. San Francisco: Jossey-Bass.

Schwartz, H. (1992). Universals in the content and structure of values: Theoretical advances and empirical tests in 20 countries. En M. P. Zanna (Ed.), Advances in experimental social psychology, 25, 1-65.

Schwartz, H. \& Jacobs, J. (2006). Sociología cualitativa: Método para la reconstrucción de la realidad. México: Trillas.

Seelos, C., \& Mair, J. (2005). Social entrepreneurship: The contribution of individual entrepreneurs to sustainable development. Bussiness Horizons, 48(3), 241-246.

Shepherd, D., \& Patzelt, H. (2011). The New Field of Sustainable Entrepreneurship: Studying Entrepreneurial Action Linking "What Is to Be Sustained" with "What Is to Be Developed. Entrepreneurship Theory and Practice, 35, 137-163.

Surin, H. (2011). Purposeful Sampling in Qualitative Research Synthesis. Qualitative Research Journal, 11(2), 6375.

Soosai-Nathan, L. (2015). Altruism: A pathway for psychological well-being. Indian Journal of Positive Psychology, 6(1), 90-92.

Stoll, M. (2015). Corporate Political Speech and Moral Obligation. Journal of Business Ethics, 123(3), 553-563.

Verissimo, M. (2008). Psicologia social \& economia solidária. [Psicología social y economia solidaria]. São Paulo, Brasil: Ideias \& Letras.

Waddock, S., \& Steckler, E. (2016). Visionaries and Wayfinders: Deliberate and Emergent Pathways to Vision in Social Entrepreneurship. Journal of Business Ethics, 133(4), 719-734.

Whittington, L. (2016). Luck, knowledge and value. Synthese, 193, 1615-1633.

Zahra, S., Gedajlovic, E., Neubaum, D., \& Shulman, J. (2009). A typology of social entrepreneurs: Motives, search processes and ethical challenges. Journal of Business Venturing, 24(5), 519-532. doi: 10.1016/j.jbusvent.2008.04.007

Zhang, D., \& Swanson, L. (2014). Linking Social Entrepreneurship and Sustainability". Journal of Social Entrepreneurship, 5(2), 175-191.

Zhao, H., \& Seibert, S. (2006). The Big Five personality dimensions and entrepreneurial status: A meta-analytical review. Journal of Applied Psychology, 91(2), 259-271. 